\title{
First report of Fusarium fujikuroi in the Lao PDR
}

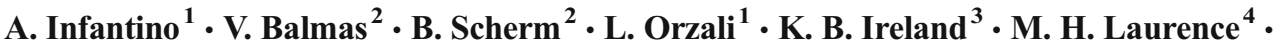 \\ S. Phantavong ${ }^{3} \cdot$ P. Phitsanoukane ${ }^{3} \cdot$ K. Vongvichid $^{3} \cdot$ S. Keopadchit ${ }^{3} \cdot$ L. W. Burgess $^{5}$
}

Received: 29 June 2016 / Accepted: 27 February 2017 / Published online: 9 March 2017

(C) Australasian Plant Pathology Society Inc. 2017

\begin{abstract}
The isolation of Fusarium fujikuroi from rice plants with elongated stems typical of bakanae disease in the Lao PDR is reported for the first time. The identification was based on both molecular and morphological markers. Koch's postulates were fulfilled. Bakanae disease has long been recognized in the Lao PDR based on symptomatology, but the etiology of the disease has not been clearly defined. This study was part of a continuing program to assist with the development of checklists of pathogens and plant diseases for Lao PDR for biosecurity purposes and integrated disease management.
\end{abstract}

Keywords Bakanae disease $\cdot$ Fusarium fujikuroi $\cdot$ Rice . Oryza sativa

Bakanae disease remains a significant problem in some rice (Oryza sativa) growing areas worldwide (Amatulli et al. 2010; Carter et al. 2008; Ou 1985). The characteristic symptom of the disease is the abnormal elongation of the stems, which is

M. H. Laurence

matthew.laurence@environment.nsw.gov.au

1 Consiglio per la Ricerca in Agricoltura e per l'analisi dell'Economia Agraria, Centro di ricerca per la patologia vegetale, Via C.G. Bertero, 22, I-00156 Rome, Italy

2 Dipartimento di Agraria, Università degli Studi di Sassari, Via E. De Nicola 9, I-07100 Sassari, Italy

3 Provincial Agriculture and Forestry, Thaluang Village, Pakse, Champasak, Lao People's Democratic Republic

4 The Royal Botanic Gardens and Domain Trust, Mrs Macquaries Rd, Sydney, NSW 2000, Australia

5 Faculty of Agriculture and Environment, The University of Sydney, Sydney 2006, Australia visible from the late seedling stage of growth. Other symptoms include a reduction in tiller number per plant, pale green flag leaves and sterile empty panicles. Crown rot and stunting of seedlings and adult plants has also been observed (Webster and Gunnell 1992). Rice cultivation in the Lao PDR can be traced back for more than 6000 years. It accounts for more than $80 \%$ of the area under cultivation within the country and for almost $80 \%$ of inhabitants' calorie in-take (Schiller et al. 2006).

In the Lao PDR, bakanae disease has not been considered economically important, as relatively low-input production systems still prevail in most rice-growing areas of the country (Schiller et al. 2001). Nevertheless, the incidence of bakanae has increased in recent years due to changes in agricultural practices, and the introduction of higher-yielding but more susceptible rice varieties (Douangboupha et al. 2006). However, the etiology of the disease has not been clearly defined, with the pathogen having been reported as Fusarium moniliforme (nomen confusem) and Gibberella fujikuroi (Douangboupha et al. 2006), with no formal reports of the isolation of Fusarium fujikuroi in the Lao PDR and no cultures deposited in an internationally recognized culture collection.

In August 2013 bakanae disease with typical elongated stems (Fig. 1a) was observed in some crops in Savannakhet, Salavan and Champasak provinces during an ad hoc rice disease survey. Crown rot symptoms were also observed in some stunted plants (Fig. 1b). Five diseased plants with typical bakanae symptoms were collected from a farm in Ponthong district, Champasak province for isolation of the pathogen.

The lower stem base of an elongated tiller was removed from each plant, and washed thoroughly with tap water. A $2 \mathrm{~cm}$ long stem section, including the second node, was excised from each stem base. These sections were rinsed in sterile water, then surface sterilized in $70 \%$ ethyl alcohol for 
Fig. 1 a Rice plants affected by bakanae disease showing typical elongation of stems in the field in Lao PDR. b Stunted rice plants affected by the crown rot symptom of bakanae disease in the Lao PDR. c Fusarium fujikuroi (ICMP 20040) Pathogenicity test: Tray with noninoculated seedlings on left, and tray with inoculated seedlings on right showing elongation of the first internode and the entire seedlings (note that dead seedlings are not visible). d Comparison of non-inoculated (left) and inoculated seedlings (right) illustrating the elongation of the first internode and the entire seedling
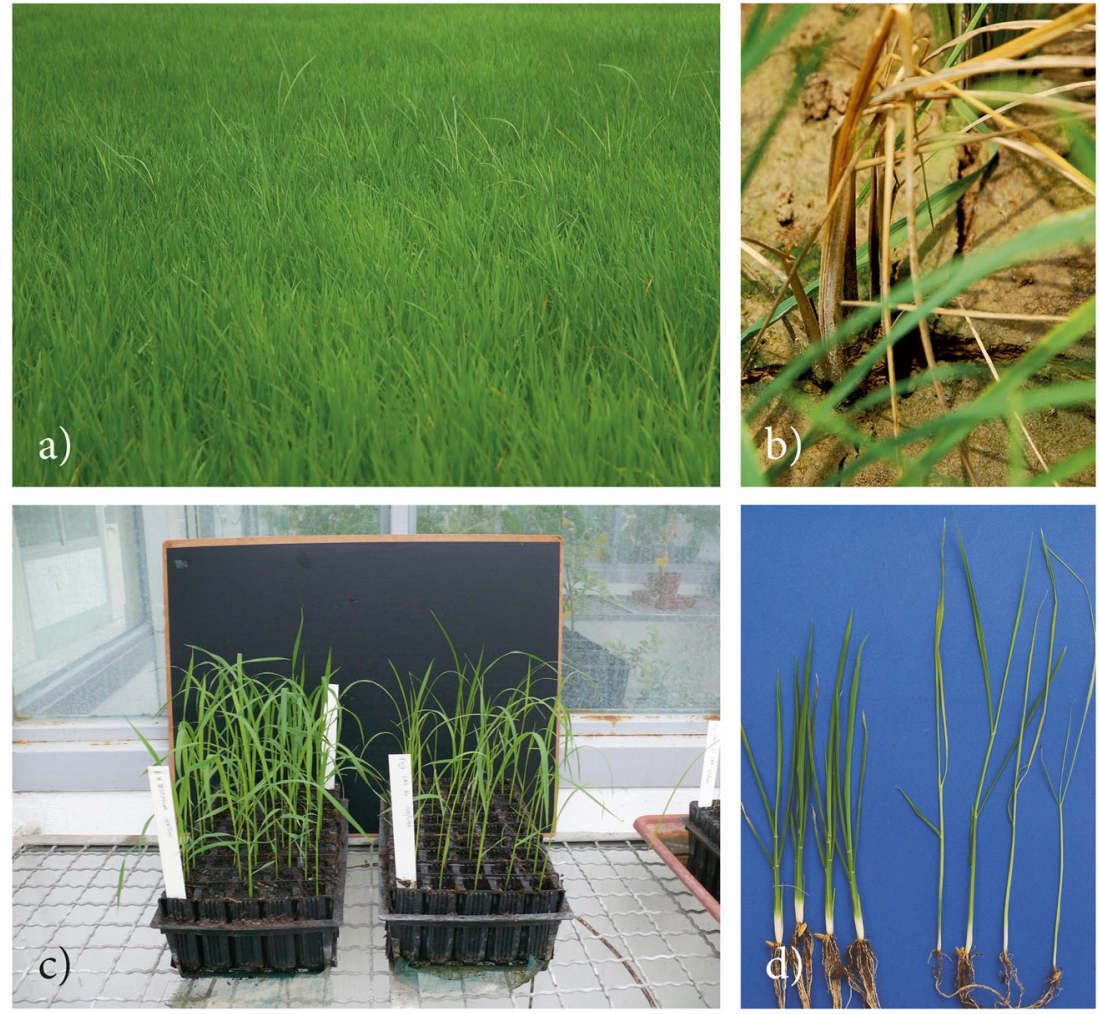

$10 \mathrm{~s}$, rinsed again in sterile water and damp-dried on sterile paper tissue. Oblique transverse stem segments were then removed from the section and plated on quarter strength potato sucrose agar ( $1 / 4 \mathrm{PSA})$. The plates were incubated under artificial light at room temperature $\left(25-30{ }^{\circ} \mathrm{C}\right)$ for $4 \mathrm{~d}$. Putative colonies of a Fusarium species developed from all segments. These were sub-cultured to carnation leaf-piece agar (CLA). Conidia from these colonies on CLA were used to establish pure cultures by single conidium transfer (Burgess et al. 1994) and grown on CLA and $1 / 4$ PSA under the conditions described above for $10 \mathrm{~d}$. These were putatively identified morphologically as Fusarium fujikuroi (Gerlach and Nirenberg 1982).

One isolate (CHA-11), was deposited in the International Collection of Microorganisms from Plants (ICMP, New Zealand) and accessioned as ICMP20040. This isolate was also sent to the Dipartimento di Agraria, Università degli Studi di Sassari, Italy for confirmation of identification by morphological and molecular methods. The pure culture of ICMP20040 was grown on Spezieller Nährstoffarmer Agar (SNA) (Nirenberg 1976) and the morphological identification was confirmed as $F$. fujikuroi based on the presence of microconidia in medium to long chains produced from monophialidic or polyphialidic conidiophores (Fig. 2a-g); microconidia in false-heads on polyphialides (Fig. 2c); the shape of macroconidia, slender almost straight, with a slightly hooked apical cell and a slightly foot shaped basal cell (Fig. 2d,e). Fusarium fujikuroi does not produce chlamydospores but some strains can form swollen cells as illustrated in Fig. $2 \mathrm{~h}$. Morphologically, F. fujikuroi resembles $F$. proliferatum, but from our experience $F$. fujikuroi produces less polyphialides on SNA, and produces pale-orange sporodochia more rapidly and abundantly (Fig. 2f).

The morphological identification was confirmed by species-specific PCR and phylogenetic analysis. DNA was extracted following the protocol of Aljanabi and Martinez (1997) from mycelium grown for $7 \mathrm{~d}$ at $25{ }^{\circ} \mathrm{C}$ on potato dextrose agar (PDA) medium. A species-specific PCR was conducted with the primer pair FujilF/TEFlR and PCR conditions described in Amatulli et al. (2010) that resulted in a positive $179 \mathrm{bp}$ band for $F$. fujikuroi. The translation elongation factor 1-alpha $(E F-1 \alpha)$ region was amplified with the primer pair EF1/EF2 and PCR conditions described in Carbone and Kohn (1999) and PCR products were sequenced at BMR Genomics (Padova, Italy) and sequence deposited in GenBank (KX432970). Identification was based on phylogenetic analysis of the $E F-1 \alpha$ gene region using unweighted parsimony, neighbour-joining and Bayesian likelihood tree construction methods. Sequences were aligned using the multiple alignment program ClustalW (Version 1.83) plug-in (Thompson et al. 1997) in the software Geneious (Version 5.4.7) (Kearse et al. 2012). The alignment was edited manually using the sequence alignment editing program Geneious (Version 5.4.7) and all polymorphisms were confirmed by reexamining the electropherograms. Parsimony analysis was conducted in PAUP 4.0b10 (Swofford 2002) using the 
Fig. 2 Morphological characteristics of Fusarium fujikuroi (ICMP 20040). (a, b, g) Microconidia in chains produced from monophialidic and polyphialidic conidiophores in culture on SNA. (c) Microconidia in false heads formed on polyphialides on SNA. (d,e) Slender, almost straight macroconidia with a slightly hooked apical cell and a slightly foot-shaped basal cell, from sporodochia on filter-paper pieces on SNA (f). (h) Swollen thin walled cells in chains in hyphae on SNA
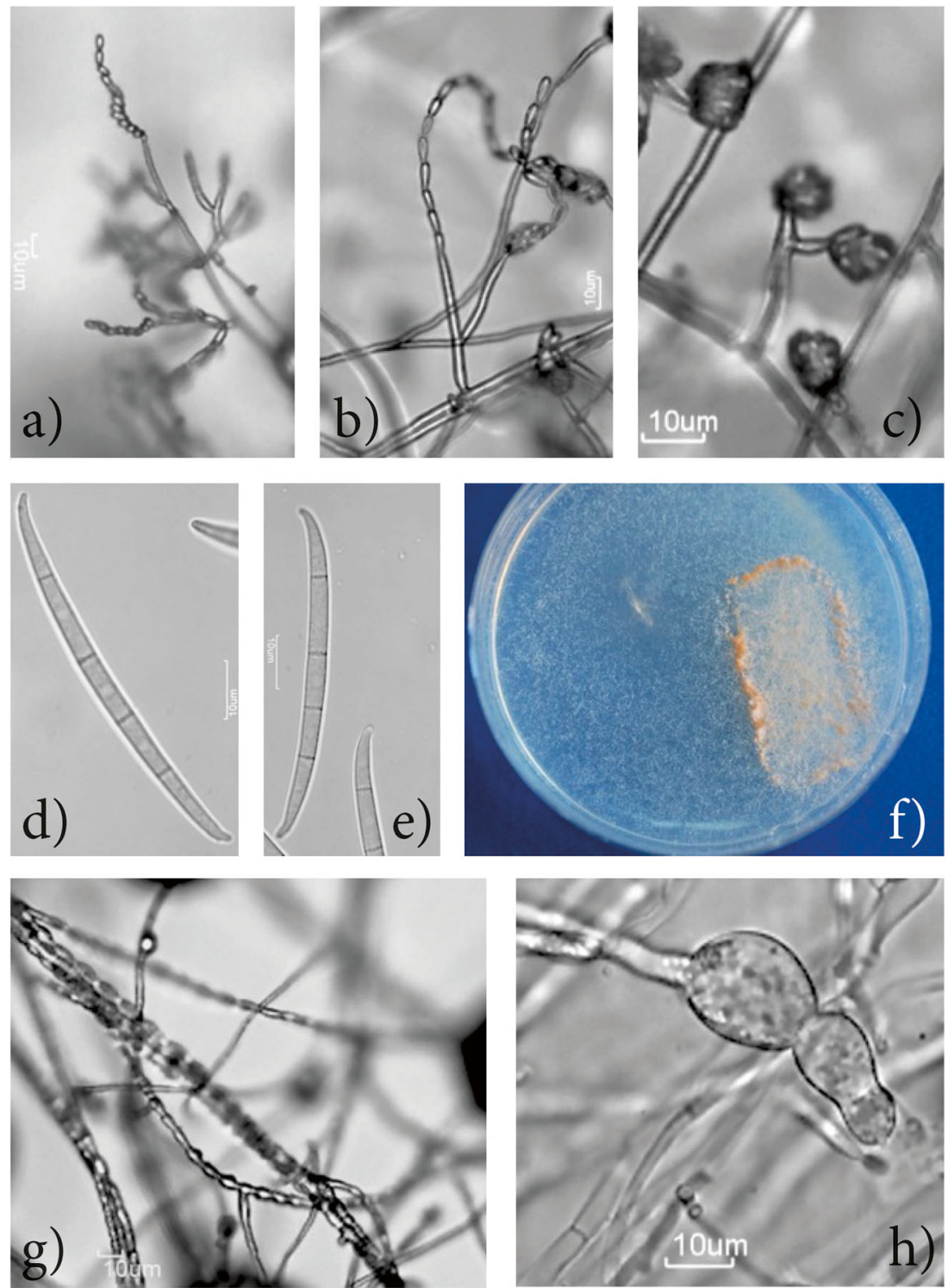

heuristic search option with 1000 random addition sequences and tree bisection reconnection branch swapping. Gaps were treated as missing data. The consistency index $(\mathrm{CI})$ and retention index (RI) were calculated to indicate the amount of homoplasy present. The $E F-1 \alpha$ data set was rooted with F. commune (NRRL22903) and F. oxysporum (NRRL22902) as these species have been established as suitable outgroup taxa for the F. fujikuroi Species Complex (O'Donnell et al. 1998). Clade stability was assessed in PAUP 4.0b10 using 1000 heuristic search bootstrap replications with random sequence addition (MPBS). Bayesian inference was used to generate posterior probabilities (PP) for consensus nodes using MRBAYES (Version 2.0.3) plug-in (Huelsenbeck 2001) in the software Geneious (Version 5.4.7) (Kearse et al. 2012). The Monte Carlo Markov Chain was run with 2,000,000 generations using the General Time Reversible (GTR) substitution model with gamma rate distribution as determined by jModelTest (Posada 2008). Trees were visualised and edited using FigTree v1.4 (Rambaut 2013).

The EF-1 $\alpha$ data set consisted of 641 nucleotides, of which 101 were parsimony-informative characters. The parsimony analysis resulted in one single most parsimonious tree $(\mathrm{CI}=0.71 \mathrm{RI}=0.76)$ (Fig. 3). The Lao isolate, ICMP20040, clustered with vouchered taxa of $F$. fujikuroi with both MPBS and PP support.

A pathogenicity test was undertaken using the susceptible rice cultivar Gange, after determining the seed was free from seed-borne $F$. fujikuroi using the deep-freezing blotter test (Limonard 1966). Fifty seeds were sown in sterilized soil in seed trays (Rootrainers ${ }^{\mathrm{TM}}$, Tildenet Group, UK) and inoculated by adding $1 \mathrm{ml}$ of conidial suspension on each seed. The conidial suspension was generated using three $5 \mathrm{~mm}$ square plugs of each isolate grown on PDA and transferred to $100 \mathrm{~mL}$ 


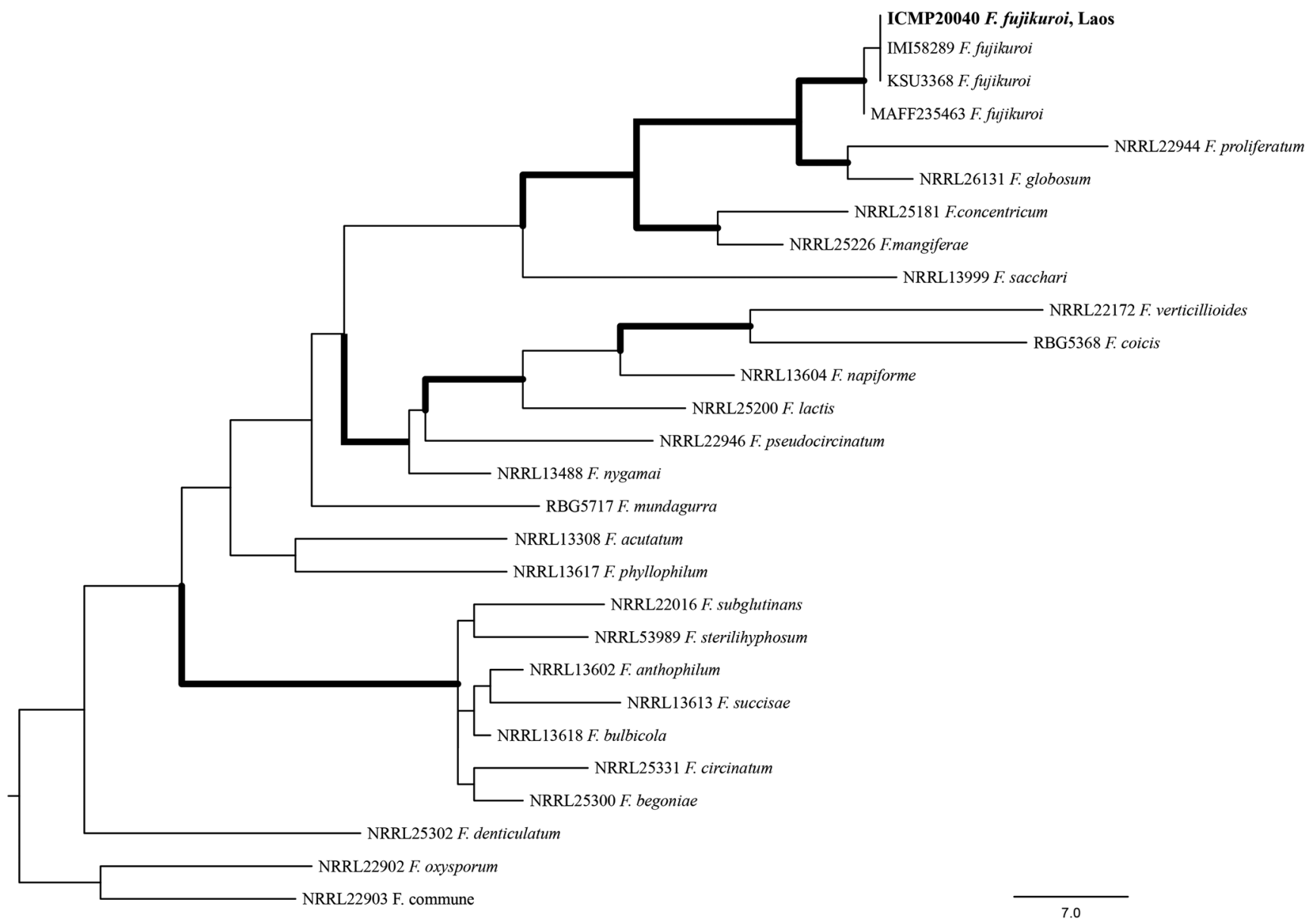

Fig. 3 One of 46 most-parsimonious trees depicting the phylogenetic relationship between the Lao PDR isolate ICMP20040, Fusarium fujikuroi sensu strictu and other species in the Fusarium fujikuroi

Czapek-Dox liquid medium in Erlenmeyer flasks and grown on a rotary shaker for $3 \mathrm{~d}$ at $120 \mathrm{rpm}$ and $25^{\circ} \mathrm{C}$. Conidia were filtered through two layers of sterile gauze, and washed twice with sterile water. Final inoculum concentration was adjusted to $1 \times 10^{6}$ spores $\mathrm{ml}^{-1}$ with sterile water. The control consisted of 100 untreated seeds. A disease scale (0-4) of Zainudin et al. (2008) was used to rate the symptoms with the following modifications: $0=$ no symptoms; $1=$ normal growth but leaves beginning to show yellowish-green, small necrosis localized at the crown level; 2 = abnormal growth, elongated, thin and yellowish-green leaves; seedlings also shorter or taller than normal, necrosis on main root and crown; $3=$ abnormal growth, elongated, chlorotic, thin and brownish leaves; seedlings also shorter or taller than normal, reduced root system with necrosis on secondary roots and on basal stem; $4=$ dead plants. A disease severity index for each isolate was generated from the scoring values using the McKinney index (I) (McKinney 1923).

The seedlings grown from inoculated seed all developed symptoms of disease by $30 \mathrm{~d}$ after planting. Some plants were elongated (Fig. 1c), others were stunted, and some had
Species Complex. Branches with most parsimonious bootstrap partitions greater than $70 \%$ and Bayesian posterior probabilities greater than 0.95 are indicated in bold

necrotic lesions at the soil line that led to seedling death. The seedlings in the control treatment did not develop symptoms (Fig. 1d). The disease severity index was $76 \%$ for inoculated plants and $0 \%$ for non-inoculated plants. Re-isolations were attempted from both inoculated and non-inoculated seedlings. Fusarium fujikuroi was only re-isolated from the diseased seedlings, fulfilling Koch's postulates.

Bakanae disease of rice has long been recognized in the Lao PDR by the conspicuous elongated stems produced by many infected plants (Douangboupha et al. 2006). In this note we report the isolation of $F$. fujikuroi from plants with typical symptoms of elongated stems and the successful fulfillment of Koch's postulates. We also observed typical crown rot symptoms in some stunted plants. This study was part of a continuing program to assist with the development of checklists of plant pathogens and diseases for biosecurity and trade purposes, as well as integrated disease management in the Lao PDR.

Acknowledgements The authors gratefully acknowledge financial support through the RISINNOVA Project, AGER (Grant 20102369), and the Crawford Fund of Australia. The authors also acknowledge the 
support provided by the Champasak and Savannakhet Provincial Agriculture and Forestry Offices. The fifth author was an Australian Volunteer for International Development, an Australian Government Program.

\section{References}

Aljanabi SM, Martinez I (1997) Universal and rapid salt-extraction of high quality genomic DNA for PCR-based techniques. Nucleic Acids Res 25:4692-4693

Amatulli MT, Spadaro D, Gullino ML, Garibaldi A (2010) Molecular identification of Fusarium spp. associated with bakanae disease of rice in Italy and assessment of their pathogenicity. Plant Pathol 59: 839-844. doi:10.1111/j.1365-3059.2010.02319.x

Burgess LW, Liddell CM, Summerell BA (1994) Laboratory manual for Fusarium research. Laboratory manual for Fusarium research, 3rd edn. University of Sydney, Sydney

Carbone I, Kohn LM (1999) A method for designing primer sets for speciation studies in filamentous ascomycetes. Mycologia 91:553556

Carter LL, Leslie JF, Webster RK (2008) Population structure of Fusarium fujikuroi from California rice and water grass. Phytopathology 98:992-998. doi:10.1094/phyto-98-9-0992

Douangboupha B, Khamphoukeo K, Inthavong S, Schiller JM, Jahn G (2006) Pests and diseases of the rice production systems of Laos. In: Schiller JM (ed) Rice in Laos. IRRI, Los Baños, Philippines, pp 265-282

Gerlach W, Nirenberg H (1982) The genus Fusarium: a pictorial atlas. Mitteilungen aus der Biologischen Bundesanstalt für Land- und Forstwirtschaft Berlin-Dahlem, Heft 209. Kommissionsverlag P. Parey, Berlin

Huelsenbeck JP (2001) MRBAYES: Bayesian inference of phylogenetic trees. Bioinformatics 17:754-755

Kearse M, Moir R, Wilson A et al (2012) Geneious basic: an integrated and extendable desktop software platform for the organization and analysis of sequence data. Bioinformatics 28:1647-1649
Limonard T (1966) A modified blotter test for seed health. Neth J Plant Pathol 72:319-321

McKinney HH (1923) Influence of soil temperature and moisture on infection of wheat seedlings by Helminthosporium sativum. J Agric Res 26:195-217

Nirenberg HI (1976) Untersuchungen über die morphologishe differenrierung in der Fusarium Sektion Liseola. Mitteilungen aus der Biologischen Bundesanstalt Für Land- und Forstwirtschaft (Berlin-Dahlem) 169:1-117

O'Donnell K, Cigelnik E, Nirenberg HI (1998) Molecular systematics and phylogeography of the Gibberella fujikuroi species complex. Mycologia 90:465-493

Ou SH (1985) Bakanae disease and foot rot. In: Ou SH (ed) Rice diseases, 2nd edn. Commonwealth Agricultural Bureau Slough, UK, pp 262 272

Posada D (2008) jModelTest: Phylogenetic Model Averaging. Mol Biol Evol 25:1253-1256. doi:10.1093/molbev/msn083

Rambaut A (2013) FigTree http://tree.bio.ed.ac.uk/software/figtree/. Accessed 6.2.2014

Schiller JM, Linquist B, Douangsila K, et al (2001) Constraints to rice production systems in Laos. In: ACIAR Proceedings. ACIAR; 1998, 3-19

Schiller JM, Chanphengxay MB, Linquist B, Appa Rao S (2006) Rice in Laos. International Rice Research Institute, Los Baños, Philippines

Swofford DL (2002) PAUP*. Phylogenetic Analysis Using Parsimony (*and Other Methods). Version 4. Sinauer Associates, Sunderland

Thompson JD, Gibson TJ, Plewniak F, Jeanmougin F, Higgins DG (1997) The CLUSTAL_X windows interface: flexible strategies for multiple sequence alignment aided by quality analysis tools. Nucleic Acids Res 25:4876-4882

Webster RK, Gunnell PS (1992) Compendium of rice diseases. APS Press, St. Paul

Zainudin N, Razak AA, Salleh B (2008) Bakanae disease of rice in Malaysia and Indonesia: etiology of the causal agent based on morphological, physiological and pathogenicity characteristics. Journal of Plant Protection Research 48:475-485 